\title{
Dynamic Distributor Routing in Supply Chain Networks with
}

\section{Stochastic Travel Time}

\author{
Ali Fakhrali ${ }^{1 *}$, Kamal Ghouchanian ${ }^{2} \&$ Sasan Siavashi ${ }^{3}$ \\ ${ }^{1}$ Kalleh Dairy Co., Tehran, Iran \\ ${ }^{2}$ University of Sistan and Baluchestan, Iran \\ ${ }^{3}$ Young researchers and Elite Club, Tehran, Iran \\ *Ali Fakhrali, E-mail: a.fakhrali@kalleh.com
}

Received: June 16, 2017

Accepted: July 8, 2017

Online Published: July 13, 2017

doi:10.22158/rem.v2n4p11

URL: http://dx.doi.org/10.22158/rem.v2n4p11

\begin{abstract}
Minimizing the distribution time in supply chain networks is critical. By minimizing the total time of distribution in the network we can reduce the cost as well as decrease the product wastage for goods with fast approaching expiration date such as dairy products. In real-world the traveling time in supply chain network is not deterministic most of the time and uncertainties in the form of randomness are not avoidable. For this reason, for finding the optimal path of distributor vehicles in the distribution network that has the lowest travel time, a probabilistic dynamic optimization model has been used in this study and the results of a numerical example are discussed.
\end{abstract}

\section{Keywords}

vehicle routing, supply chain, network optimization

\section{Introduction and Background}

There are some concerns that affect designing a supply chain network such as reducing operation cost while improving quality and speed of service and minimizing the product wastage. Another goal in the supply chain network design that tremendously affects the performance of the system is to have a proper risk management procedure. Decision making for risk management consists of determining role of technology, demand prediction, error reduction, controlling economic, social, and environmental performance of the supply chain (Osvald \& Stirn, 2008). Thus taking the customer needs and demand into account and improving timely access to services while using new technologies not only reduce the risk and indirect costs of operation, but also generate more business opportunities in the competing market (Aguwa, Olya, \& Monplaisir, 2017).

Investigating the shortest path and minimizing the travel times are among the important topics which have always received significant attention in different fields of studies, especially in supply chain and 
transportation engineering. Some seminal studies provide different approaches for solving the shortest path problem (Bazaraa, Jarvis, \& Sherali, 2011; Bellman, 1958). From the viewpoint of transportation area, the traffic has a significant effect on vehicle routing problems when the main goal is finding the route with fastest travel time. There are several studies conducted in the field of traffic management and optimization (Baykaso \& Subulan, 2016; Bell, 2004; Fu \& Rilett, 1998; Lin, Nozick, \& Turnquist, 2006; Murthy \& Sarkar, 1996; Nepal, Park, \& Choi, 2009; Tsao \& Lu, 2012). Ziliaskopoulos and Mahmassani (1996) analyzed the impact of turning directions of vehicles on travel time in urban areas with high traffic volume and they found the effect significantly. PC simulation packages became more popular in the field of transportation due to the complex environment of traffic variables such as driver behavior modeling. Simulation is used in different aspects of transportation from the researches about effect of vehicle dynamic on highway systems see (Abdi \& Mehrara, 2015; Molan \& Abdi Kordani, 2014; Molan \& Kordani, 2014; Stine, Hamblin, Brennan, \& Donnell, 2010), to the studies related to the shortest travel time of routes (Abdi Kordani et al., 2014).

Incorporating technologies such as using Radio Frequency Identification technology (RFID) or GPS into the supply chain networks, specifically in food distribution systems has been widely used in recent years. Both mentioned technologies help to track vehicles and goods accurately. So, it can be beneficial for decision makers and distribution planning experts to find certain distribution patterns to plan and predict the vehicle routing. In addition, RFID systems help to track short shelf-life products (Kärkkäinen, 2003). Also, using GPS-equipped vehicles helps to get almost an accurate understanding of routes and travelling time estimation. However, assessing the impact and operating cost of using new technologies as well as setting standards for using them is essential for improving the supply chain development and reducing the costs such as energy and product wastage costs.

Recently there are lots of effort to design green supply chains. Efficient eco-friendly supply chain design emerges as a mechanism to obviate global warming and human health issues that are caused by greenhouse gas emissions and air pollution of transportation systems. One way to make the distribution systems more efficient is to minimize the distance and traveling time of the vehicles because longer distances have a negative effect on the environment (Elhedhli \& Merrick, 2012).

To address above-mentioned concerns and issues, we focused on finding the shortest path in the supply chain network. Researchers used different optimization methods to solve the shortest path problem including exact, heuristic and meta-heuristics approaches (Binart, Dejax, Gendreau, \& Semet, 2016; Lopes, Barreto, Ferreira, \& Santos, 2008; Mohammed et al., 2017; Oyola, Arntzen, \& Woodruff, 2016; Tarantilis \& Kiranoudis, 2001; Wu, Low, \& Bai, 2002). By using a stochastic dynamic programming method, we can maintain the quality of the product while minimizing the travel time of distributor vehicles. We model the distribution network between the factory, distribution centers and retailers as a dynamic shortest path problem with time-dependent travel times where a distribution has been fitted on each arc length in the network. The objective is to find the optimal route that has shortest travel time. This objective can indirectly lead to increased customer satisfaction, maintaining the quality of the 
product, reduced product waste and operation cost.

As mentioned, finding the shortest path for delivering products is very significant due to the high cost that imposed on the supply chain network as wastage. There are different methods listed in the literature review for finding the shortest path but most of them use the expected value of random variables instead of using the density functions directly (Fu \& Rilett, 1998; Issac \& Campbell, 2015; Orda \& Rom, 1990). One of the recent works in finding the shortest path in networks with random variable arc lengths is conducted by Olya, Shirazi and Fazlollahtabar (2013). They proposed a stochastic dynamic programing to find the shortest path where travel times are normal random variables. They used different algorithms such as dynamic programming and Dijkstra's algorithm to solve the stochastic model (Olya, 2014a). Finding the shortest path in other types of networks where the travelling time of arcs have various arc lengths is proposed by (Olya, 2014b). The proposed method uses convolution and dynamic and probability theory for adding the travel times and finding the minimum density function in each stage of dynamic programming (Olya, Fazlollahtabar, \& Mahdavi, 2013). Since this method is dynamic, there is no need to calculate the total travel time for each step, so this method performs faster than other methods. Also, in their studies the randomness is considered with not using the expected value instead of the distribution arc length for each link in the network. This method helps to find the shortest path in ground and air transportation as well as supply chain networks and automated guided vehicle equipped manufacturing systems (Fazlollahtabar \& Olya, 2013).

The rest of this paper is organized as follows: Section 2 describes the problem, model and the adapted method for solving the problem. In section 3, a case study is presented and after applying the method on the data, the results are compared with process simulation results and we will show how to implement the stochastic dynamic programming method proposed by Olya and Fazlollahtabar (2014) for finding the shortest route in the supply chain network. Eventually, the last section includes conclusions and directions for future research.

\section{Stochastic Supply Chain Modeling}

Consider a supply chain network as it is shown in Figure 1. This network consists of a finite set of stakeholders (nodes) such as factories distribution centers, retailers (customers) and wastage warehouse as well as finite numbers of arcs that represent the transportation links between each node. These nodes and arcs form a directed acyclic network. So, it is accepted to assume that each path starting from the origin (factory) to the destination (wastage warehouse) is always continuous as we move from left to right.

In this study, as it is mentioned in the previous section, for the sake of considering uncertainty in the model instead of considering the traveling time as a deterministic value, the travelling time is assigned by a specific distribution for each pair of nodes. The length of each arc is either an exponential random variable with parameter $\lambda_{i}$, a gamma random variable with shape parameter $\alpha$ and rate parameter $\lambda_{j}$ or a 
normal random variable with mean $\mu$ and standard deviation $\sigma$.

Consider that distributor vehicles start from the factory by collecting the final products which are dairy products in this study that need to be delivered very fast. Then the product is delivered to the distribution centers. After arranging the products for delivery to retailers the distributor vehicle delivers the product to retailers and collects their wastage from previous orders and transfers them to the wastage ware house to be processed for recycling or emission. The objective is to minimize the total span time of distribution vehicle in the supply chain network and find the shortest route in terms of traveling time, from the source node to the sink node. This helps to improve the quality, making the SCM more efficient and reducing the cost of delivery process and energy consumption. To obtain the optimum route of the network a stochastic dynamic method proposed by Olya and Fazlollahtabar (2014) has been used in this study due to its computation speed and efficiency. Also this method offers a probabilistic and dynamic solution for finding the shortest path which is essential in diminution industries.

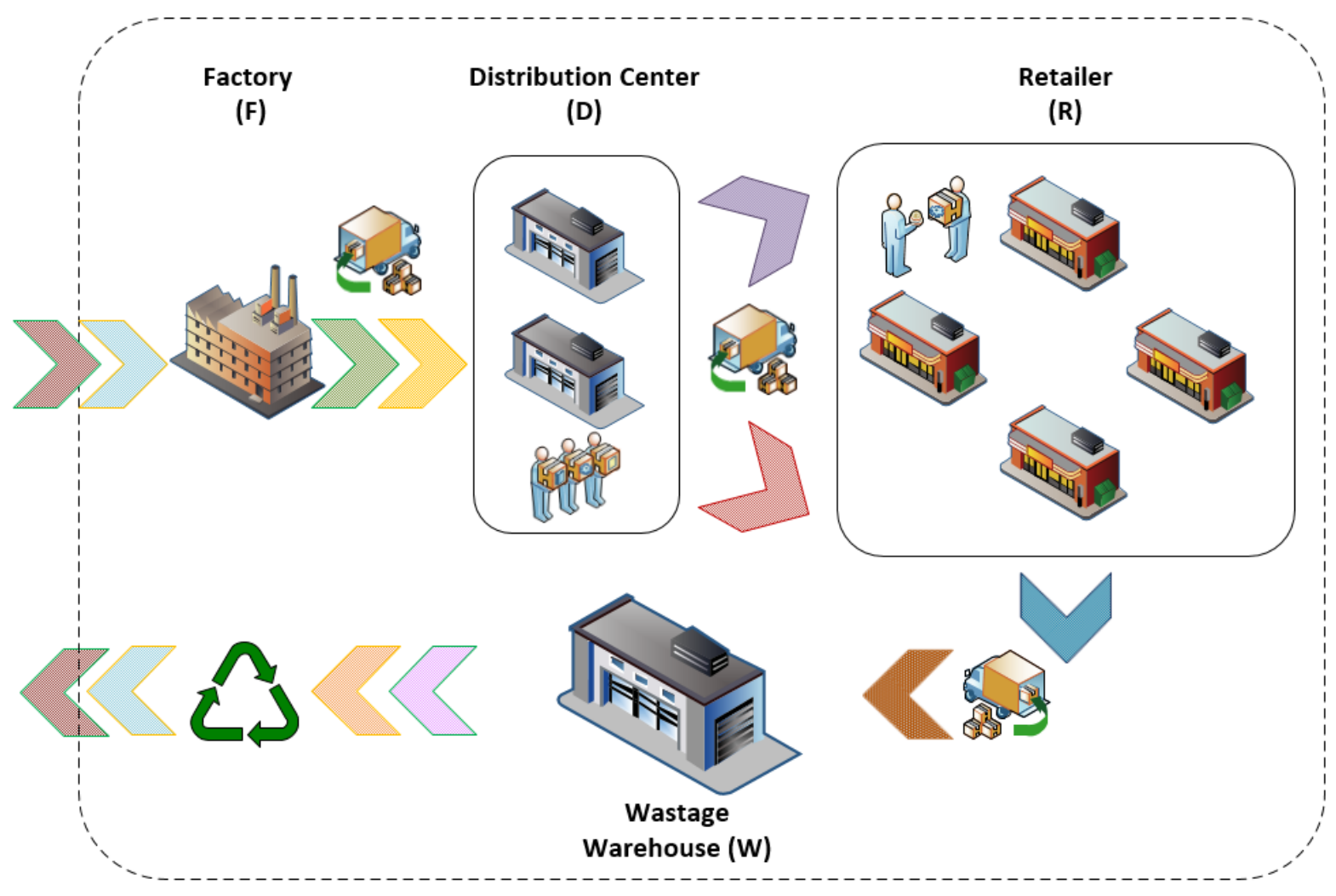

Figure 1. A Distribution Network with Multiple Service Providers

The proposed method uses the backward dynamic programming approach to find the shortest path. But since in this study the traveling time has been considered as a random variable with certain distributions, we need to define a mechanism for aggregating each distribution to find the total travellingtime; then we will be able to compare the total time of each route in each stage of dynamic 
programming. To define the optimal value function, we should consider the following: This function represents the distribution of the shortest path from a certain node to the destination node. Also, $d_{i j}$ represents the distance between each pair of nodes $i$ and $j$. Thus, the optimal value function $R_{i}$ can be defined as:

$R_{i}=$ the density function of the shortest path from stake holder $i$ to node $\mathrm{N}$.

Suppose that there are $\mathrm{N}$ stakeholders in the supply chain network which is an acyclic network. Equation 1 defines the recurrence relation in stage number $i$.

$$
R_{i}=\min _{j>i}\left\lfloor d_{i j}+R_{j}\right\rfloor \text { for } i=\mathrm{N}-1, \ldots 1
$$

To initiate the backward dynamic programming the boundary condition is defined as $R_{N}=0$.

As we mentioned, every step of dynamic programming consists of aggregating the total traveling time. So, since the traveling times are not deterministic values, here we use convolution to find the distribution of sum of two traveling time density function in each stage.

Theorem 1: consider density functions $f_{X}(x)$ and $f_{Y}(y)$ of two continuous random variables $X$ and $Y$, respectively where $f_{X}(x)$ and $f_{Y}(y)$ are defined for all real numbers. Then the density function of summation of two random variable $X$ and $Y$ is a random variable $z$ with density function $f_{Z}(z)$, where $f_{Z}$ is the convolution of $f_{X}$ and $f_{Y}$ (Olya \& Fazlollahtabar, 2014).

$$
f z(\mathrm{z})=\int_{-\infty}^{+\infty} f_{X}(X) f_{y}(z-x) d x=\int_{-\infty}^{+\infty}(z-y) f_{Y}(y) d y
$$

After finding the total time of traveling from each node to the destination in each step, the backward dynamic programming method requires us to compare the total time of each node and choose the minimum value. So, we need to find the probability that one probability density function becomes smaller than the other probability density function. To calculate the probability of the random variable $X_{I}$ being smaller than the second random variable $X_{2}$, equation 3 is used.

$$
P\left(X_{1}<X_{2}\right)=\int_{0}^{\infty} \int_{x_{1}}^{\infty} f_{x 2}\left(x_{2}\right) \cdot f_{x 1}\left(x_{1}\right) d x_{2} d x_{1}
$$

In the next section of the paper, we have studied a real issue in the dairy industry by applying the described method. We have considered the travelling time as a random variable due to uncertainty in the model caused by some factors that affect traveling time such as, road traffic, weather condition, unexpected stops and crashes in the route, inconsistency inhuman-related work performance, etc. The result of this approach has been compared to the simulation result in the next section.

\section{Case Study Analysis and Results}

In this section, we present the implementation of the described method. A dataset of GPS-equipped vehicles traveling times has been used, the data of travelling time between each pair of nodes have been collected. Then a distribution function for each arc lengths has been fitted to various probability density 
functions including exponential, gamma and normal density functions. The distribution network is depicted in Figure 1 which includes factory, distribution centers, retailers and wastage warehouse. The objective is to find the best route for a distributor vehicle that has the minimum travelling time. Let us define each of mentioned parts of network as stages. We have 4 stages in the network. The networks are denoted as F: Factory, D: Distribution center, R: Retailer and W: Wastage warehouse. So, by considering 1 origin and 1 distention, there would be 1 node in each of the stages $\mathrm{F}$ and $\mathrm{W}$. We consider 2 nodes for each of the middle stages $\mathrm{D}$ and $\mathrm{R}$. The length of connecting arcs has been represented in Table 1.

Table 1. Travelling Time Distribution

\begin{tabular}{llllllll}
\hline Stage & Node & 1 & 2 & 3 & 4 & 5 & 6 \\
\hline F & 1 & - & $\mathrm{N}(30.2,2.1)$ & $\mathrm{N}(40.8,1.2)$ & & - & - \\
$\mathrm{D}$ & 2 & - & - & - & $\mathrm{G}(8.4,2.2)$ & $\operatorname{Exp}(13.7)$ & - \\
$\mathrm{D}$ & 3 & - & - & - & $\mathrm{G}(12.8,1.6)$ & $\operatorname{Exp}(18.5)$ & - \\
$\mathrm{R}$ & 4 & - & - & - & - & - & $\mathrm{Exp}(4.3)$ \\
$\mathrm{R}$ & 5 & - & - & - & - & - & $\mathrm{G}(8.4,2.3)$ \\
$\mathrm{W}$ & 6 & - & - & - & - & - & - \\
\hline
\end{tabular}

A distributor vehicle starts from node 1 in stage factory, and can choose to go to either node 2 or 3 in stage $\mathrm{D}$, then form the selected node in stage $\mathrm{D}$, it can choose to go to either node 4 or 5 to deliver the product, then after collecting the product waste of previous orders from retailers, it goes to node 6 in stage $\mathrm{W}$. The backward search starts with the boundary condition: $\mathrm{R}_{6}=0$.

Considering the probability density functions presented in Table 1 and the described methodology in section 3, the results of the shortest path in each stage as well as the probability of having the minimum travelling time for each path are shown in Table 2.

Table 2. Backward Minimum Path Dynamic Search

\begin{tabular}{lll}
\hline State & Path & Prob. \\
\hline S6 & 6 & 1 \\
S5 & $5-6$ & 1 \\
S4 & $4-6$ & 1 \\
S3 & $3-4-6$ & 0.643 \\
S2 & $2-4-6$ & 0.749 \\
S1 & $1-2-4-6$ & 0.866 \\
\hline
\end{tabular}

As presented in Table 2, each node has been represented by a state number S and the shortest path of the supply chain network is the path 1-2-4-6. This path ensures going through each stage and product delivery to one stakeholder at a time. The probability that the path is minimal is 0.866 . In order to 
validate the result of this solution, we used process simulation. The result of the simulation is represented in Table 3.

Table 3. Simulated Traveling Time for Every Path in the Network

\begin{tabular}{llll}
\hline Path & Average & Minimum & Maximum \\
\hline $1-2-4-6$ & 35.681 & 32.554 & 39.219 \\
$1-2-5-6$ & 46.286 & 40.371 & 52.932 \\
$1-3-4-6$ & 57.216 & 51.882 & 63.433 \\
$1-3-5-6$ & 62.704 & 56.958 & 68.295 \\
\hline
\end{tabular}

The total travelling time of each possible path has been calculated using the simulation study. As it is represented in the Table above, the simulation results validate the previous results from the adapted dynamic programming method.

\section{Conclusion and Recommendations}

In this study, we used a stochastic dynamic programming method to model and solve a vehicle routing problem in adistribution network where the traveling times are independent random variables instead of being deterministic values. The results of the study determine the shortest path along with its probability of being the path with minimum traveling time. As mentioned in the paper, the performance of the solution method is validated by simulation results. This method has less cost due to being dynamic, so there is no need to calculate the total time of all available paths. Improving the speed in distribution networks is of high importance because it can help to preserve the product quality. A proposed future work can be considering the capacity of each node while delivering the products in the network so the model can be solved as a multi-objective optimization problem.

\section{References}

Abdi Kordani, A., \& Mehrara Molan, A. (2015). The effect of combined horizontal curve and longitudinal grade on side friction factors. KSCE Journal of Civil Engineering, 19(1). https://doi.org/10.1007/s12205-013-0453-3

Abdi Kordani, A., Mehrara Molan, A., \& Monajjem, S. (2014). New Formulas of Side Friction Factor Based on Three-Dimensional Model in Horizontal Curves for Various Vehicles. In T\&DI Congress 2014: Planes, Trains, and Automobiles (pp. 592-601). https://doi.org/10.1061/9780784413586.057

Aguwa, C., Olya, M. H., \& Monplaisir, L. (2017). Modeling of fuzzy-based voice of customer for business decision analytics. Knowledge-Based Systems, 125, 136-145. https://doi.org/10.1016/j.knosys.2017.03.019

Baykaso lu, A., \& Subulan, K. (2016). A multi-objective sustainable load planning model for 
intermodal transportation networks with a real-life application. Transportation Research Part E: Logistics and Transportation Review, 95, 207-247.

Bazaraa, M. S., Jarvis, J. J., \& Sherali, H. D. (2011). Linear programming and network flows. John Wiley \& Sons.

Bell, M. G. H. (2004). Games, heuristics, and risk averseness in vehicle routing problems. Journal of $\begin{array}{llll}\text { Urban Planning } & \text { 37-41. }\end{array}$ https://doi.org/10.1061/(ASCE)0733-9488(2004)130:1(37)

Bellman, R. (1958). On a routing problem. Quarterly of Applied Mathematics, 16(1), 87-90. https://doi.org/10.1090/qam/102435

Binart, S., Dejax, P., Gendreau, M., \& Semet, F. (2016). A 2-stage method for a field service routing problem with stochastic travel and service times. Computers and Operations Research, 65, 64-75. https://doi.org/10.1016/j.cor.2015.07.001

Elhedhli, S., \& Merrick, R. (2012). Green supply chain network design to reduce carbon emissions. Transportation Research Part D: Transport and Environment, 17(5), 370-379. https://doi.org/10.1016/j.trd.2012.02.002

Fazlollahtabar, H., \& Olya, M. H. (2013). A cross-entropy heuristic statistical modeling for determining total stochastic material handling time. The International Journal of Advanced Manufacturing Technology, 67(5-8), 1631-1641. https://doi.org/10.1007/s00170-012-4596-z

$\mathrm{Fu}$, L., \& Rilett, L. R. (1998). Expected shortest paths in dynamic and stochastic traffic networks. Transportation Research Part B: Methodological, 32(7), 499-516. https://doi.org/10.1016/S0191-2615(98)00016-2

Issac, P., \& Campbell, A. M. (2015). Shortest path problem with arc failure scenarios. EURO Journal on Transportation and Logistics, 1-25.

Kärkkäinen, M. (2003). Increasing efficiency in the supply chain for short shelf life goods using RFID tagging. International Journal of Retail \& Distribution Management, 31(10), 529-536. https://doi.org/10.1108/09590550310497058

Lin, J.-R., Nozick, L. K., \& Turnquist, M. A. (2006). Strategic design of distribution systems with economies of scale in transportation. Annals of Operational Research, 144, 161-180. https://doi.org/10.1007/s10479-006-0004-0

Lopes, R. B., Barreto, S., Ferreira, C., \& Santos, B. S. (2008). A decision-support tool for a capacitated location-routing problem. Decision Support Systems, 46(1), 366-375. https://doi.org/10.1016/j.dss.2008.07.007

Mohammed, M. A., Ghani, M. K. A., Hamed, R. I., Mostafa, S. A., Ibrahim, D. A., Jameel, H. K., \& Alallah, A. H. (2017). Solving Vehicle Routing Problem by Using Improved K-Nearest Neighbor Algorithm for Best Solution. Journal of Computational Science. https://doi.org/10.1016/j.jocs.2017.04.012

Molan, A. M., \& Abdi Kordani, A. (2014). Multi-Body simulation modeling of vehicle skidding and Published by SCHOLINK INC. 
roll over for horizontal curves on longitudinal grade. In 93rd Annual Meeting of the Transportation Research Board. Washington, DC.

Molan, A. M., \& Kordani, A. A. (2014). Optimization of speed hump profiles based on vehicle dynamic performance modeling. Journal of Transportation Engineering, 140(8). https://doi.org/10.1061/(ASCE)TE.1943-5436.0000686

Murthy, I., \& Sarkar, S. (1996). A relaxation-based pruning technique for a class of stochastic shortest path problems. Transportation Science, 30(3), 220-236. https://doi.org/10.1287/trsc.30.3.220

Nepal, K. P., Park, D., \& Choi, C.-H. (2009). Upgrading arc median shortest path problem for an urban transportation network. Journal of Transportation Engineering, 135(10), 783-790. https://doi.org/10.1061/(ASCE)0733-947X(2009)135:10(783)

Olya, M. H. (2014a). Applying Dijkstra's algorithm for general shortest path problem with normal probability distribution arc length. International Journal of Operational Research, 21(2), 143. https://doi.org/10.1504/IJOR.2014.064541

Olya, M. H. (2014b). Finding shortest path in a combined exponential-gamma probability distribution arc length. International Journal of Operational Research, 21(1), 25. https://doi.org/10.1504/IJOR.2014.064020

Olya, M. H., \& Fazlollahtabar, H. (2014). Finding Shortest Path in a Combined Exponential-Gamma-Normal Probability Distribution Arc Length. Advances in Industrial Engineering and Management, 3(4), 35-44.

Olya, M. H., Fazlollahtabar, H., \& Mahdavi, I. (2013). Shortest Path Problem with Gamma Probability Distribution Arc Length. International Journal of Applied Operational Research, 2(4), 55-66. Retrieved from http://www.ijorlu.liau.ac.ir/article-1-208-en.html

Olya, M. H., Shirazi, B., \& Fazlollahtabar, H. (2013). Adapted Dynamic Program to Find Shortest Path in a Network having Normal Probability Distribution Arc Length. Advances in Industrial Engineering and Management, 2(1), 5-10.

Orda, A., \& Rom, R. (1990). Shortest-path and minimum-delay algorithms in networks with time-dependent edge-length. Journal of the ACM (JACM), 37(3), 607-625. https://doi.org/10.1145/79147.214078

Osvald, A., \& Stirn, L. Z. (2008). A vehicle routing algorithm for the distribution of fresh vegetables and similar perishable food. Journal of Food Engineering, 85(2), 285-295. https://doi.org/10.1016/j.jfoodeng.2007.07.008

Oyola, J., Arntzen, H., \& Woodruff, D. L. (2016). The stochastic vehicle routing problem, a literature review, 33-65.

Stine, J. S., Hamblin, B. C., Brennan, S. N., \& Donnell, E. T. (2010). Analyzing the influence of median cross-section design on highway safety using vehicle dynamics simulations. Accident Analysis \& Prevention, 42(6), 1769-1777. https://doi.org/10.1016/j.aap.2010.04.018

Tarantilis, C. D., \& Kiranoudis, C. T. (2001). A meta-heuristic algorithm for the efficient distribution of Published by SCHOLINK INC. 
perishable foods. Journal of Food Engineering, 50(1), 1-9. https://doi.org/10.1016/S0260-8774(00)00187-4

Tsao, Y., \& Lu, J. (2012). A supply chain network design considering transportation cost discounts. Transportation Research Part E, 48(2), 401-414. https://doi.org/10.1016/j.tre.2011.10.004

Wu, T., Low, C., \& Bai, J. (2002). Heuristic solutions to multi-depot location-routing problems. $\begin{array}{llll}\text { Computers } \quad \& \quad \text { Operations } & \text { 1393-1415. }\end{array}$ https://doi.org/10.1016/S0305-0548(01)00038-7

Ziliaskopoulos, A. K., \& Mahmassani, H. S. (1996). A note on least time path computation considering delays and prohibitions for intersection movements. Transportation Research Part B: Methodological, 30(5), 359-367. https://doi.org/10.1016/0191-2615(96)00001-X 